\title{
User-Oriented Graph Visualization Taxonomy: A Data-Oriented Examination of Visual Features
}

\author{
Kawa Nazemi, Matthias Breyer, and Arjan Kuijper \\ Fraunhofer Institute for Computer Graphics Research, \\ Fraunhofer Str. 5, 64283 Darmstadt, Germany \\ \{kawa.nazemi, matthias.breyer, arjan.kuijper\} @igd.fraunhofer.de
}

\begin{abstract}
Presenting information in a user-oriented way has a significant impact on the success and comprehensibility of data visualizations. In order to correctly and comprehensibly visualize data in a user-oriented way data specific aspects have to be considered. Furthermore, user-oriented perception characteristics are decisive for the fast and proper interpretation of the visualized data. In this paper we present a taxonomy for graph visualization techniques. On the one hand it provides the user-oriented identification of applicable visual features for given data to be visualized. On the other hand the set of visualization techniques is enclosed which supports these identified visual features. Thus, the taxonomy supports the development of user-oriented visualizations by examination of data to obtain a beneficial association of data to visual features.
\end{abstract}

Keywords: graph visualization taxonomy, user-oriented visualization, visual features.

\section{Introduction}

Information Visualization aims to provide visualization techniques to present data in an efficient and effective way [11]. As main objective the comprehensive preprocessing of the data can be named, so users can immediately gather information and interact with it to identify relevant aspects. The evolved visualization techniques in the Information Visualization community utilize different visualization metaphors to present the data and impart implied data characteristics to the user. These metaphors, in turn, utilize different visual features, like color, order, size, shape, etc., to gain their metaphoric expressiveness.

The purposeful usage of visual features depends on two factors: On the one hand an appropriate mapping of data characteristics to visual features is crucial. Visual features vary in their suitability for presenting and imparting specific data, which depends on the kind of given data characteristics. On the other hand visual features are interpreted by users in different ways, thus the resulting mental map in mind of the user may strongly differ from the intention the visualization designer aspired. As a matter of fact different visualization techniques support diverse sets of visual features. Thus, the varying support for visual features and the varying suitability of visual features for specific data characteristics leads to the following, scarcely contradicted statement: different visualization techniques differ in their suitability for presenting different data characteristics. 
In this paper we present a new user-oriented taxonomy of graph visualization techniques based on the data-oriented analysis of supported visual features. The taxonomy classifies the visualization techniques according to commonly supported visual features. Furthermore, the taxonomy implies a selection guide based on the given data characteristics for an appropriate mapping of data to visual features. The selection guide offers a sequence of the compatible visual feature according to their clearness and directness for the user. So the taxonomy can be used to identify appropriate graph visualization techniques depending on the data characteristics to be visualized.

The graph visualization taxonomy is the result of a three-parted process. In Section 2 the first process step dealing with identifying the visual variables is described. In the second process in Section 3 a survey of graph visualization techniques is presented which summarizes and arranges the visualization techniques into user oriented clusters. The outcomes of steps one and two are the input for process step three, described in Section 4. Here user oriented graph visualization taxonomy based on the visual features will be constructed. The last Section demonstrates how this taxonomy can be utilized to bridge the gap between data characteristics and human perception characteristics.

\section{Fundamental Visual Features}

To display information in an intuitive manner and thus impart knowledge to users visual features (or visual variables) are utilized. These visual features act like graphical metaphors which point out the information itself in a way which structures the visual communication. Each visual feature reflects perception characteristics which include regularities for cognitive processing [18]. To obtain an overview of visual features some related work is described here.

In the research field of Information Visualization visual features had been examined for a long time. The most prominent and fundamental works are these of Bertin [3] and Mackinlay [16]. In their observation they found out these visual features radically effect humans' perception of the displayed information. So they identified visual features and chunked them into reasonable groups.

\subsection{Visual Variables}

Bertin brought up the theory of visual variables in his work semiology of graphics [3]. This theory claims that these variables fundamentally impacts the way of perceiving graphically displayed information. Therefore he enlists the following visual variables: position, shape, orientation, color, motion, texture, value, and size where the variable value can be interpreted as luminance or grey tone to distinguish it from the variable color. These variables had been differentiated into the four categories associative, selective, ordered/ordering, and quantitative visual variables. In Fig. 1 these visual variables are graphically presented. 


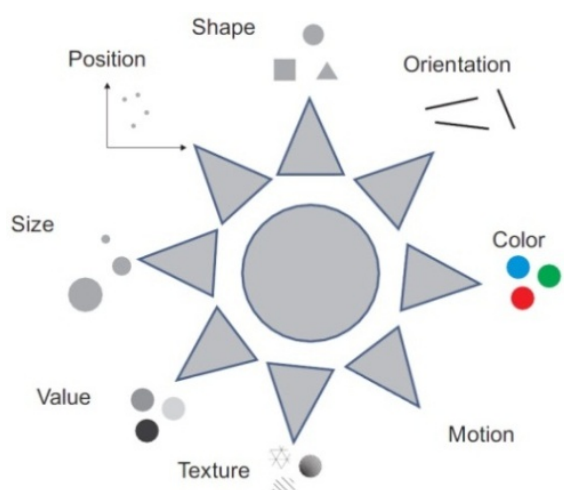

Fig. 1. Visual variables by Bertin [3], taken from [16]

\subsection{Ranking of Perceptual Tasks for Visual Variables}

Based on the work described in Section 2.1 Mackinlay extended these visual variables by length, angle, volume, connection, and containment [15]. Furthermore, he identified a ranking of perceptual tasks for the sets of quantitative, ordinal and nominal visual variables. These rankings are displayed in Fig. 2.

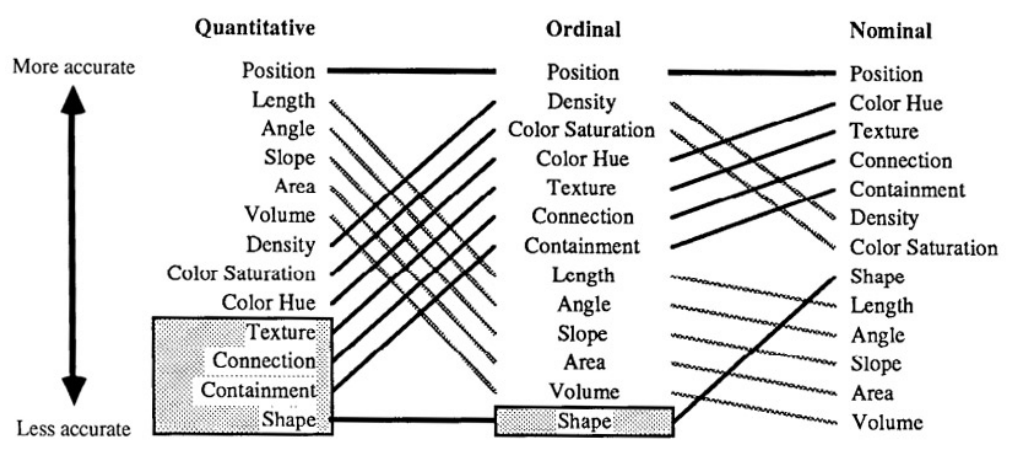

Fig. 2. Ranking of perceptual tasks for visual variables [15]

\section{Graph Layout Techniques}

For the hierarchy of visualization techniques two-dimensional graph-based layouts are examined. These are the most adequate visualization techniques for the standard user: he can easily interact with them and most accurately interpret the displayed data.

The identification of supplied visual variables in a graph visualization technique are examined according to these variables. Most existing graph technique surveys differentiate according to mathematical, behavioral, or perceptual (like visual clutter) aspects. For the approach presented in this paper a user-specific differentiation according to aspects of the visual result is required. 


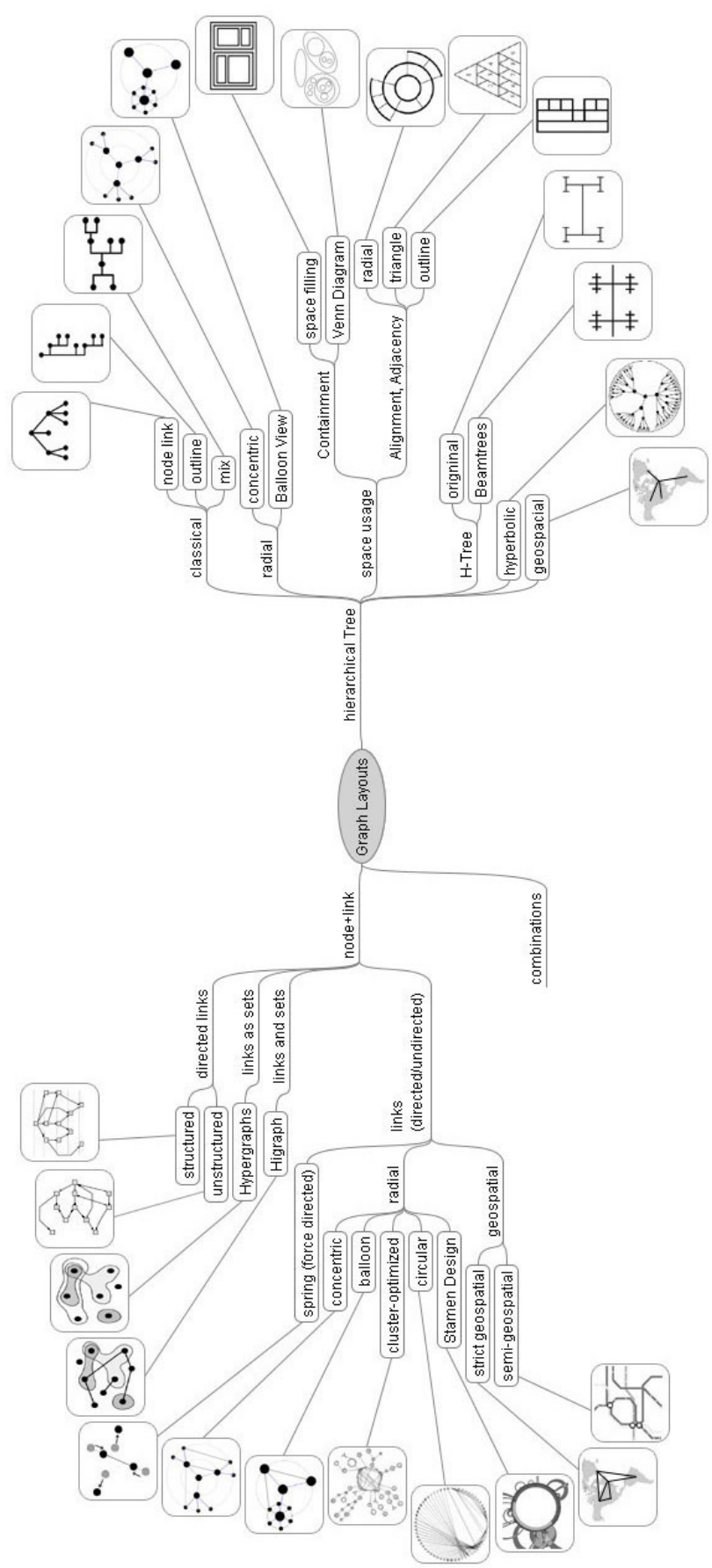

Fig. 3. User-oriented structure of graph layout visualization techniques 
Therefore, existing surveys [4,5,8,10,12,17,19] were examined and restructured. The taxonomical differentiation is based on Keim [10,12] and is transformed to differentiate the visual result the user perceives $[7,13]$. To obtain a most complete picture of visualization techniques also some important works examining specialized techniques are regarded $[1,2,6,9,14]$.

As a results of the visualization technique survey, the taxonomy (shown in Fig. 3) differentiates on the first level in i) tree layouts, ii) network layouts, and iii) combinations, which mainly depends on the given data structure to visualize. The second level differentiates predominantly according to the visual result the user perceives, which correlates with the set of supported visual variables.

\section{User-Oriented Taxonomy of Graph Visualizations}

In this Section the graph layout techniques (see Section 3) are analyzed according their support of visual features (Section 2.). The techniques are evaluated according the visual variables they support.

\subsection{Support for Visual Features in Visualization Techniques}

The evaluation of the support of specific visual features in the visualization techniques is presented in Fig. 4. If a feature is available in the visualization it is marked as ' $\mathrm{X}$ ', if not it is marked as '-'. If a variable is included but not designated for displaying information but could principally be utilized for this it is marked as ' $(\mathrm{X})$ ', if a variable is not available in a graph layout but could be integrated it is marked as '(-)'.

\begin{tabular}{|c|c|c|c|c|c|c|c|c|c|c|c|c|c|c|c|c|c|}
\hline \multirow{3}{*}{$\begin{array}{l}\text { visual variable } \\
\text { Position }\end{array}$} & \multicolumn{8}{|c|}{ hierarchical Tree } & \multicolumn{9}{|c|}{ node+link } \\
\hline & 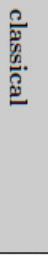 & : & 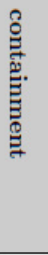 & 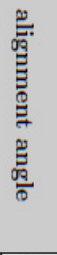 & . & 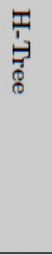 & 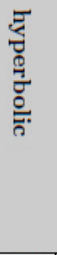 & 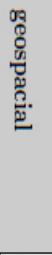 & 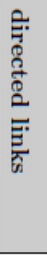 & 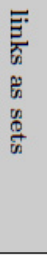 & 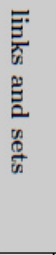 & 莺 & 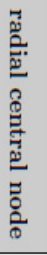 & 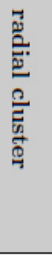 & 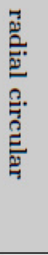 & 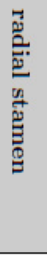 & 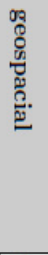 \\
\hline & (x) & - & - & $(\mathrm{x})$ & $(\mathrm{x})$ & $(\mathrm{x})$ & $(\mathrm{x})$ & $*_{-}$ & $\mathrm{x}$ & $\mathrm{x}$ & $\mathrm{x}$ & $(\mathrm{x})$ & - & $\mathrm{x}$ & - & - & $*_{-}$ \\
\hline Length & $\mathrm{x}$ & - & - & - & - & $\mathrm{x}$ & $\mathrm{x}$ & $*_{-}$ & $\mathrm{x}$ & - & $(\mathrm{x})$ & $(\mathrm{x})$ & $(-)$ & $(-)$ & $(-)$ & $(-)$ & $*_{-}$ \\
\hline Angle & $(\mathrm{x})$ & $(\mathrm{x})$ & - & $\mathrm{x}$ & - & - & $(\mathrm{x})$ & - & $\mathrm{x}$ & - & $(\mathrm{x})$ & $(-)$ & $(\mathrm{x})$ & (x) & (x) & $(\mathrm{x})$ & - \\
\hline Orientation & $(-)$ & - & (x) & - & - & - & $(\mathrm{x})$ & - & $(\mathrm{x})$ & - & $(\mathrm{x})$ & $(-)$ & - & - & - & - & - \\
\hline Size & $\mathrm{x}$ & $\mathrm{x}$ & $\mathrm{x}$ & $\mathrm{x}$ & $\mathrm{x}$ & $\mathrm{x}$ & $\mathrm{x}$ & $\mathrm{x}$ & $\mathrm{x}$ & $\mathrm{x}$ & $\mathrm{x}$ & $\mathrm{x}$ & $\mathrm{x}$ & $\mathrm{x}$ & $\mathrm{x}$ & $\mathrm{x}$ & $\mathrm{x}$ \\
\hline Saturation & $\mathrm{x}$ & $\mathrm{x}$ & $\mathrm{x}$ & $\mathrm{x}$ & $\mathrm{x}$ & $\mathrm{x}$ & $\mathrm{x}$ & $\mathrm{x}$ & $\mathrm{x}$ & $\mathrm{x}$ & $\mathrm{x}$ & $\mathrm{x}$ & $\mathrm{x}$ & $\mathrm{x}$ & $\mathrm{x}$ & $\mathrm{x}$ & $\mathrm{x}$ \\
\hline Color & $\mathrm{x}$ & $\mathrm{x}$ & $\mathrm{x}$ & $\mathrm{x}$ & $\mathrm{x}$ & $\mathrm{x}$ & $\mathrm{x}$ & $\mathrm{x}$ & $\mathrm{x}$ & $\mathrm{x}$ & $\mathrm{x}$ & $\mathrm{x}$ & $\mathrm{x}$ & $\mathrm{x}$ & $\mathrm{x}$ & $\mathrm{x}$ & $\mathrm{x}$ \\
\hline Texture & - & - & $\mathrm{x}$ & $\mathrm{x}$ & $\mathrm{x}$ & $\mathrm{x}$ & - & $(\mathrm{x})$ & - & $\mathrm{x}$ & $\mathrm{x}$ & - & - & - & - & $\mathrm{x}$ & $(\mathrm{x})$ \\
\hline Relations & - & - & - & - & - & - & - & - & $\mathrm{x}$ & $\mathrm{x}$ & $\mathrm{x}$ & $\mathrm{x}$ & $\mathrm{x}$ & $\mathrm{x}$ & $\mathrm{x}$ & $\mathrm{x}$ & $\mathrm{x}$ \\
\hline Containment & - & - & $\mathrm{x}$ & - & - & - & - & $(\mathrm{x})$ & - & $\mathrm{x}$ & $\mathrm{x}$ & - & - & - & - & - & $(\mathrm{x})$ \\
\hline Shape & $\mathrm{x}$ & $\mathrm{x}$ & - & - & - & $\mathrm{x}$ & $\mathrm{x}$ & $\mathrm{x}$ & $\mathrm{x}$ & $\mathrm{x}$ & $\mathrm{x}$ & $\mathrm{x}$ & $\mathrm{x}$ & $\mathrm{x}$ & $\mathrm{x}$ & $\mathrm{x}$ & $\mathrm{x}$ \\
\hline Motion & - & $(-)$ & - & - & - & - & $(-)$ & - & - & $(-)$ & $(-)$ & $\mathrm{x}$ & $(-)$ & $(-)$ & $(-)$ & $(-)$ & - \\
\hline Hierarchy & $\mathrm{x}$ & $\mathrm{x}$ & $\mathrm{x}$ & $\mathrm{x}$ & $\mathrm{x}$ & $\mathrm{x}$ & $\mathrm{x}$ & $(\mathrm{x})$ & $\mathrm{x}$ & $(-)$ & $(\mathrm{x})$ & $\mathrm{x}$ & $\mathrm{x}$ & - & - & - & $(\mathrm{x})$ \\
\hline
\end{tabular}

Fig. 4. Evaluation of support for visual features in the graph layout visualization techniques 


\subsection{Procedure for Construction of Graph Visualization Taxonomy}

Based on the evaluation of support for visual features in the graph layouts in Fig. 4 and the feature classes quantitative, ordinal, and nominal by Mackinlay [16] (Section 2.2), the user-oriented taxonomy is build up. In this paper for each of these classes a single taxonomy is build up. Therefore, the following procedure is applied:

The taxonomy starts with the element all. Visual features which are supported by all techniques are assigned to this element. Afterwards, the features are to arrange according to the ranking (for quantitative, ordinal, or nominal, Section 2.2). The subsequent procedure has to be iterated until all layout techniques are included in the taxonomy, respectively each taxon at the end of this differentiation contains exactly one visualization technique. Techniques are grouped as taxon in the taxonomy which supports the first visual variable. The same holds for the techniques which do not support this visual feature. For both taxa those visual features are assigned to the taxa which are supported or not supported by all techniques included in the taxa.

\subsection{The Resulting Oser-Oriented Graph Visualization Taxonomy}

Applying the procedure for constructing, described in Section 4.2, the result is a taxonomy of graph visualization techniques. Due to the data-oriented ordering of the visual features in the procedure according to user-oriented perception of information displayed with the visual features, this resulting taxonomy is user-oriented based on the data-oriented examination of these features.

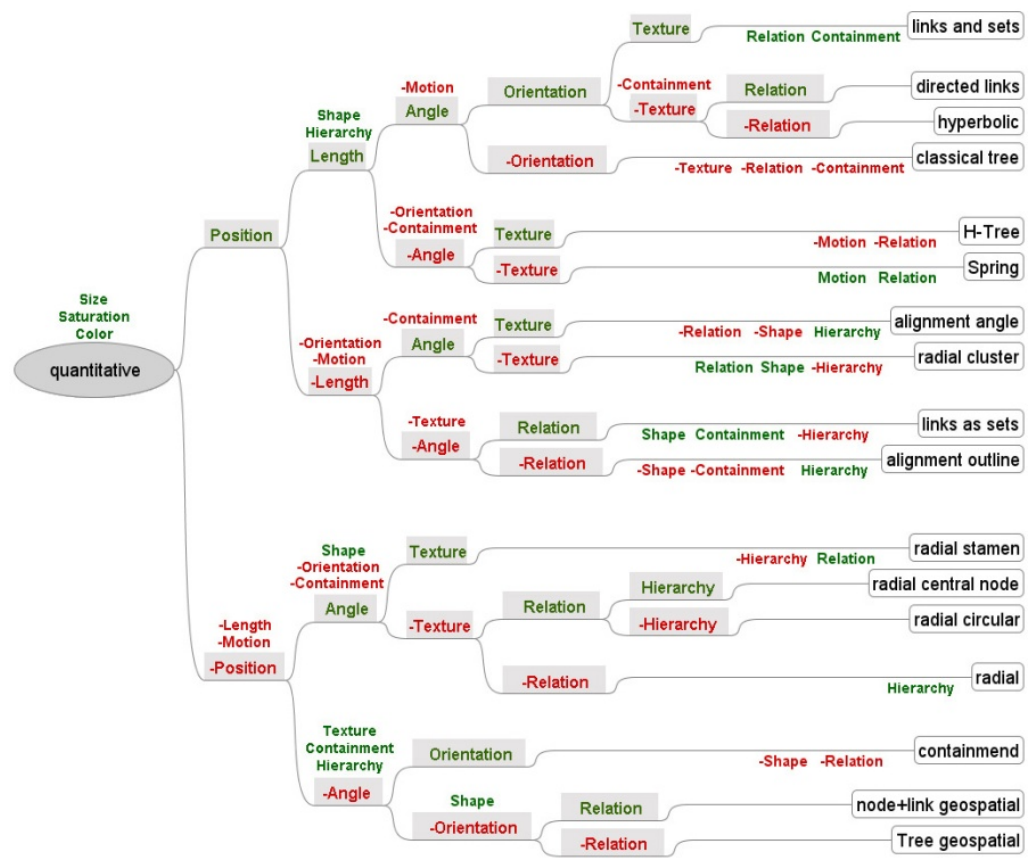

Fig. 5. Graph visualization taxonomy according to visual variables applicable for quantitative data 


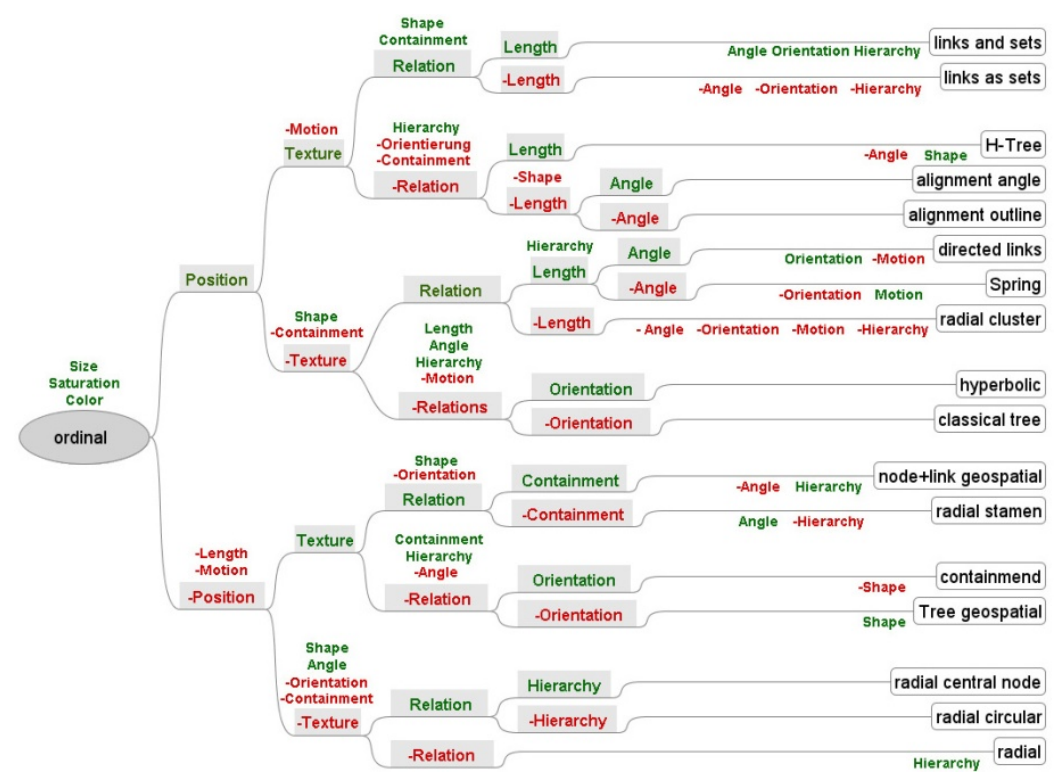

Fig. 6. Graph visualization taxonomy according to visual variables applicable for ordinal data

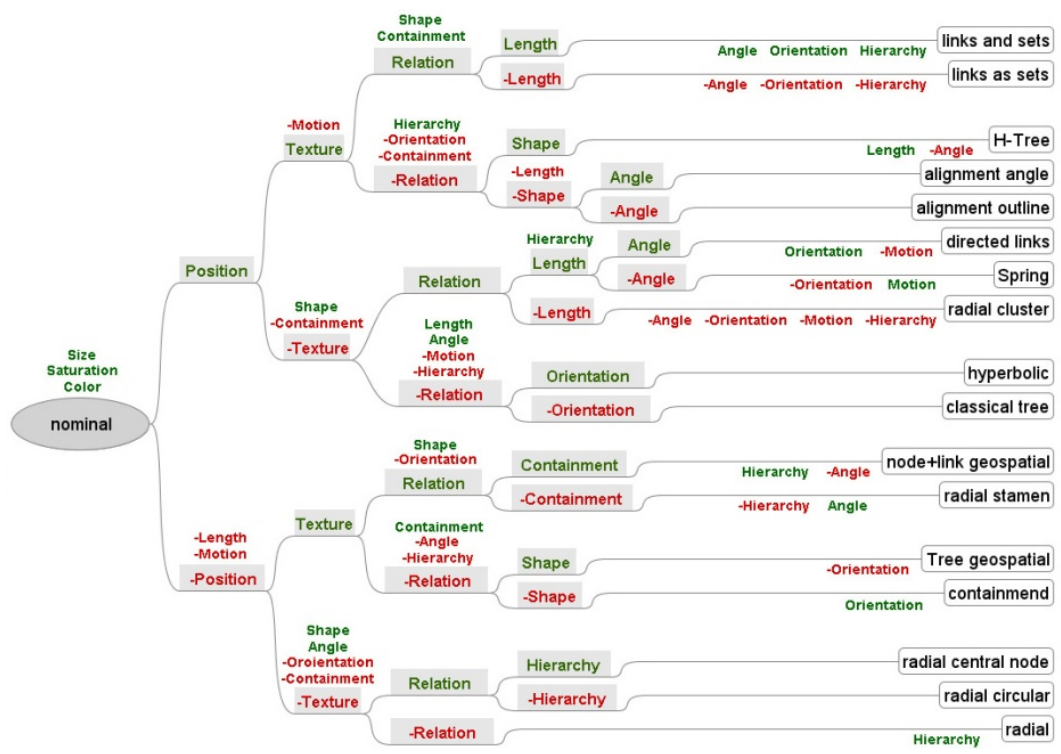

Fig. 7. Graph visualization taxonomy according to visual variables applicable for nominal data

The user-oriented taxonomies for presenting quantitative, ordinal, and nominal data with adequate visual features will be presented in the Figs. 5, 6 and 7. In these figures green colored features are available, red colored (and signed) features are not available for the specific taxon. Subsequent a guide is given how these taxonomies 
can be interpreted and be utilized to identify adequate visualization techniques for given kinds of data.

\section{Application Scenario Using the Taxonomy}

The taxonomy implies a correlation between data types and user-adequate visualization features. Thus, when the task is to present a specific data type the taxonomy depicts this visualization feature which should be utilized to visualize the data. At the same time the taxonomy presents the set of graph layout techniques which embody selected variables, if interpreted as decision tree. Thus it can be used to identify those layout techniques which can be applied to display an amount of data types.

Furthermore, on the right side the taxonomy demonstrates a sequence for the visualization techniques according to their expressiveness in terms of the number of available visual features for a given kind of data type. Thus, at the upper side this layout technique is presented which embodies most visual features. Walking down the graph layout techniques their expressiveness according to the embodied visual features decreases.

For example, when three data elements are to visualize which are of the type quantitative: According to the quantitative taxonomy when selecting the 'positive' paths in the tree the data should be visualized by utilizing position, length and angle. Furthermore, the set of graph layout techniques embodying these features are beneath the taxon 'angle'('links and sets', 'directed links', 'hyperbolic', and 'classical tree'). One of these techniques can be used to visualize the data in a user-oriented way utilizing those visual features. The final selection of the specific technique is a design decision and depends on the usage scenario and specific user group. Thus, the taxonomy preselects applicable layout techniques and provides the allocation of beneficial visual features for presenting the data that way the user can intuitively and easy percept and interact with.

\section{Conclusion}

In this paper a novel taxonomy is developed which offers the identification of adequate visual features for presenting data in a user-oriented way. To achieve this, existing visualization techniques are summarized and re-organized to a user-driven categorization. For the purpose of visualizing data for the standard user those visualization techniques were considered which are graph-based and two-dimensional. The visualization techniques were evaluated for their support of visual features. Based on the evaluation result the taxonomy had been constructed for quantitative, ordinal and nominal data types.

Using the taxonomy adequate visual features are depicted which should be utilized to visualize a given data element according to the elements data type. Furthermore, the set of visualization techniques is enclosed which support the identified visual features, which can thus be applied to display an amount of given data in a useroriented way. In addition, the taxonomy presents a sequence for the visualization techniques according to their expressiveness in term of the number of supported features. 
Due to the data type-specific ordering of the visual features according to their perception characteristics for users, thus forming a user-oriented sequence, the result is a user-oriented taxonomy for graph visualization techniques based on a data-oriented examination of visual features in these visualization techniques.

Acknowledgments. The here described work was developed as a part of the CoreTechnology-Cluster for Innovative User Interfaces and Visualizations of the THESEUS Program, a 60-month program partially funded by the German Federal Ministry of Economics and Technology.

\section{References}

1. Daniel, A., Munzner, T., Auber, D.: Topo-Layout: Multilevel Graph Layout by Topological Features. IEEE Transactions on Visualization and Computer Graphics 13(2), 305-317 (2007)

2. Wilhelm, B., Jünger, M., Mutzel, P.: Simple and Efficient Bilayer Cross Counting. In: Goodrich, M.T., Kobourov, S.G. (eds.) GD 2002. LNCS, vol. 2528, pp. 130-141. Springer, Heidelberg (2002)

3. Bertin, J.: Semiology of graphics. University of Wisconsin Press (1983)

4. Brodbeck, D., Mazza, R., Lalanne, D.: Interactive Visualization - A Survey. In: Lalanne, D., Kohlas, J. (eds.) Human Machine Interaction. LNCS, vol. 5440, pp. 27-46. Springer, Heidelberg (2009)

5. Collins, C.: CSC 2524: Graph Visualizations, 2006. Department of Computer Science, University of Toronto (2006)

6. Consens, M.P., Mendelzon, A.O.: Hy+: A Hygraphbased Query and Visualization System. In: Visualization System (Video Demonstration). Proc. ACM SIGMOD 1993, pp. 511-516 (1993)

7. Harel, D.: On Visual Formalisms. Communications of the ACM 31 (1988)

8. Herman, I., Melancon, G., Marshall, M.S.: Graph visualization and navigation in information visualization: A survey. IEEE Transactions on Visualization and Computer Graphics 6(1), 24-43 (2000)

9. Holten, D.: Hierarchical Edge Bundles: Visualization of Adjacency Relations in Hierarchical Data. IEEE Transactions on Visualization and Computer Graphics 12(5), 741-748 (2006)

10. Keim, D.A.: Databases and Visualization. In: Jagadish, H.V., Mumick, I.S. (eds.) SIGMOD Conference, p. 543. ACM Press, New York (1996)

11. Keim, D.A.: Information Visualization and Data Mining. IEEE Transactions on Visualization and Computer Graphics 7(1) (2002)

12. Keim, D.A.: Visual techniques for exploring databases. Invited Tutorial. In: Int. Conference on Knowledge Discovery in Databases, KDD 1997 (1997)

13. Kosara, R., Hauser, H., Gresh, D.: An Interaction View on Information Visualization. In: Proceedings of Eurographics (2003)

14. Leissler, M.: A Generic Framework for the Development of 3D Information Visualisation Applications. Doctor thesis, Technical University Darmstadt, Germany (2004)

15. Mackinlay, J.: Automating the Design of Graphical Presentations of Relational Information. ACM Transactions on Graphics 5, 110-141 (1986)

16. Qeli, E.: Information Visualization Techniques for Metabolic Engineering. Doctor thesis, Philipps-University Marburg, Germany (2007) 
17. Shi, K., Irani, P., Li, B.: An Evaluation of Content Browsing Techniques for Hierarchical Space-Filling Visualizations. In: INFOVIS 2005: Proceedings of the 2005 IEEE Symposium on Information Visualization, p. 11. IEEE Computer Society, Washington, DC (2005)

18. Tversky, A.: Features of Similarity. Psychological Review 84(2), 327-352 (1977)

19. Yee, K.-P., Fisher, D., Dhamija, R., Hearst, M.: Animated Exploration of Dynamic Graphs with Radial Layout. In: INFOVIS 2001: Proceedings of the IEEE Symposium on Information Visualization 2001 (INFOVIS 2001), p. 43. IEEE Computer Society, Washington, DC (2001) 